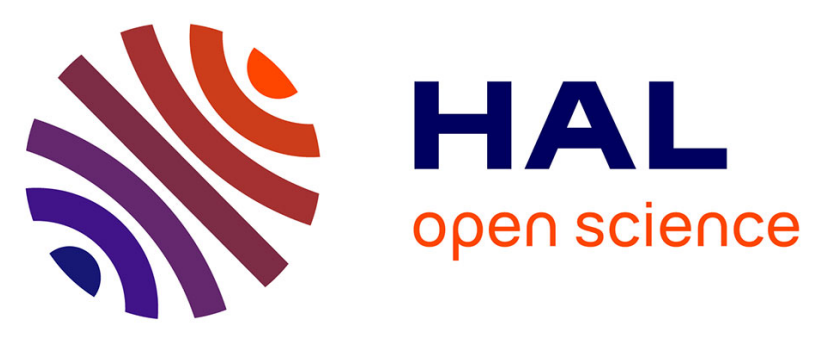

\title{
Pour les nématiques biaxes
}

\author{
G. Toulouse
}

\section{To cite this version:}

G. Toulouse. Pour les nématiques biaxes. Journal de Physique Lettres, 1977, 38 (2), pp.67-68. 10.1051/jphyslet:0197700380206700 . jpa-00231327

\section{HAL Id: jpa-00231327 https://hal.science/jpa-00231327}

Submitted on 1 Jan 1977

HAL is a multi-disciplinary open access archive for the deposit and dissemination of scientific research documents, whether they are published or not. The documents may come from teaching and research institutions in France or abroad, or from public or private research centers.
L'archive ouverte pluridisciplinaire HAL, est destinée au dépôt et à la diffusion de documents scientifiques de niveau recherche, publiés ou non, émanant des établissements d'enseignement et de recherche français ou étrangers, des laboratoires publics ou privés. 


\title{
POUR LES NÉMATIQUES BIAXES
}

\author{
G. TOULOUSE
}

Laboratoire de Physique de l'Ecole Normale Supérieure, 24, rue Lhomond, 75231 Paris, France

(Reçu le 19 novembre 1976, accepté le 7 décembre 1976)

\begin{abstract}
Résumé. - Les prédictions théoriques pour les défauts des cristaux liquides du type nématique biaxe suggèrent des propriétés inusuelles : des lignes de disclination qui se combinent comme des quaternions, c'est-à-dire de manière non commutative. Il s'ensuit une rigidité topologique qui mérite une étude expérimentale.
\end{abstract}

\begin{abstract}
Theory predicts that the defects in liquid criystals of biaxial nematic type have some unusual properties : disclination lines would combine like quaternions, i.e. in a non commutative way. This leads to a topological rigidity which merits an experimental study.
\end{abstract}

Les nématiques usuels (uniaxes) sont des cristaux liquides formés de molécules allongées alignées, les centres de gravité restant désordonnés : le paramètre d'ordre est alors un quadrupole de révolution. On peut aussi imaginer une phase où des molécules anisotropes (en forme de $T$ par exemple) aligneraient leurs différents axes, les centres de gravité restant toujours désordonnés; le paramètre d'ordre serait alors un quadrupole à trois axes inégaux; une telle phase serait, par définition, nématique biaxe.

A ma connaissance, il n'existe pas, à ce jour, de publications annonçant l'observation et la caractérisation d'une phase nématique biaxe. Il se peut que de telles phases existent naturellement (par exemple dans des milieux biologiques) sans avoir été encore reconnues. Même si ce n'est pas le cas, on peut envisager la synthèse chimique de nouvelles molécules appropriées [1]. Voici maintenant des prédictions théoriques sur la nature des défauts dans les nématiques biaxes; cette nature est si inhabituelle qu'on espère ainsi stimuler un effort expérimental pour vérifier ou infirmer ces prédictions.

Connaissant le paramètre d'ordre d'une phase ordonnée, il est possible de déterminer les défauts stables, par un critère topologique [2]. A chaque défaut stable (on entend par défaut une configuration où le paramètre d'ordre s'annule dans une certaine région, appelée cœur du défaut) est associé un invariant topologique. L'algèbre de ces invariants gouverne les règles d'association des défauts.

Prenons quelques exemples. Dans un superfluide tel que l'hélium 4, les défauts stables sont des lignes de vortex et l'invariant topologique est un nombre entier positif ou négatif; la loi de combinaison est l'addition; en termes mathématiques, on a

$$
\pi_{1}(\mathrm{~V}) \simeq \mathrm{Z}
$$

où $\pi_{1}$ signifie premier groupe d'homotopie (caractérisant les défauts de dimension $(d-2)$, donc les lignes pour un échantillon tridimensionnel), $\mathrm{V}$ est la variété des états internes (c'est-à-dire l'ensemble des orientations possibles du paramètre d'ordre, ici $\mathrm{V}=\mathrm{S}_{1}$ ) et $\mathrm{Z}$ est, par définition, le groupe additif des entiers.

Pour un superfluide tel que la phase A de l'hélium 3, le paramètre d'ordre est un trièdre [3], on $\mathrm{a} \mathrm{V}=\mathrm{SO}(3)$, le groupe des rotations à 3 dimensions et

$$
\pi_{1}(\mathrm{~V}) \simeq \mathrm{Z}_{2},
$$

où $Z_{2}$ est, par définition, le groupe additif des entiers modulo 2. Il s'ensuit qu'il existe un type de défaut linéaire stable, qui est son propre antidéfaut, la loi de combinaison étant l'addition modulo 2 [4].

Dans le premier exemple, l'invariant topologique est du type charge électrique; dans le second exemple, il est du type parité. Dans les deux cas, on a affaire à un groupe commutatif et même cyclique.

Il existe quantité d'autres exemples physiques connus. Cependant, dans les cas usuels, on a affaire à des groupes du type précédent ou à des produits de tels groupes. Il est vrai que dans le cas des défauts des systèmes périodiques, compte tenu des symétries de translation et de rotation, Louis Michel a montré qu'il pouvait exister une structure très riche [5], mais les dislocations de rotation sont en général des défauts de grande énergie et peu maniables. Dans ces 
conditions, le système le plus simple présentant des propriétés inusuelles est le nématique biaxe. Dans ce cas, on trouve en effet que

$$
\pi_{1}(\mathrm{~V}) \simeq \mathrm{Q}
$$

où $\mathrm{Q}$ est le groupe discret, à 8 éléments, non cyclique et non commutatif, appelé communément groupe des quaternions.

Les éléments du groupe $Q$ sont, hormis l'identité I, les éléments $\mathrm{e}_{1}, \mathrm{e}_{2}, \mathrm{e}_{3}$ correspondant aux rotations de $\pi$ autour des trois axes inégaux du quadrupole, leurs inverses $\left(-\mathrm{e}_{1}\right),\left(-\mathrm{e}_{2}\right),\left(-\mathrm{e}_{3}\right)$, et un élément $\mathbf{J}$ correspondant à une rotation de $2 \pi$ autour d'un axe quelconque. Les lois de combinaison sont

$$
\begin{gathered}
\mathrm{e}_{1}^{2}=\mathrm{e}_{2}^{2}=\mathrm{e}_{3}^{2}=\mathrm{J} \\
\mathrm{J}^{2}=\mathrm{I} \\
\mathrm{e}_{1} \mathrm{e}_{2}=-\mathrm{e}_{2} \mathrm{e}_{1}=\mathrm{e}_{3} \\
\mathrm{e}_{1} \mathrm{e}_{2} \mathrm{e}_{3}=\mathrm{J} .
\end{gathered}
$$

Ces résultats mathématiques sont rigoureux. Il s'agit maintenant de comprendre les conséquences physiques d'une loi non commutative.

Nous avions fait la conjecture qu'une telle loi pouvait interdire le croisement simple de deux lignes de défaut. Cette conjecture physique a été récemment examinée du point de vue mathématique par V. Poénaru [6]. Sur un modèle topologique qui représente de manière fidèle la réalité physique, il a pu démontrer un théorème qui va bien dans le sens de cette conjecture, et qui va beaucoup plus loin en précisant les obstructions topologiques au croisement. Ce théorème est démontré pour toute loi non commutative.

Dans le cas particulier des nématiques biaxes, on peut essayer d'aller plus loin et de prédire l'évolution de deux lignes poussées l'une vers l'autre mais à qui il est interdit de se croiser. Supposons ainsi que nous ayons une ligne verticale de caractère $e_{3}$ et une ligne horizontale de caractère $e_{1}$ et supposons que par quelque moyen nous soyons capables de faire déplacer la ligne $e_{1}$ dans le plan horizontal de manière à provoquer une collision avec la ligne $e_{3}$. Si le croisement simple est interdit en raison de l'obstruction topologique, on peut imaginer que le système des deux lignes suive l'évolution suivante. La ligne $e_{3}$ reconnaît que la ligne $\mathrm{e}_{1}$ n'est rien d'autre qu'une combinaison $\mathrm{e}_{2} \mathrm{e}_{3}$; elle se coupe en deux, débobine la ligne $e_{1}$ en deux brins de caractère $e_{2}$ et $e_{3}$, coupe en deux le brin $e_{3}$ et se recombine moitié à moitié avec lui. Lorsque la ligne $e_{1}$ poursuit sa progression horizontale, le débobinage se poursuit, le brin $\mathrm{e}_{2}$ joignant les deux points de débobinage s'allongeant.

$\mathrm{Au}$ lieu d'un croisement simple, on aurait ainsi une configuration enchevêtrée (bien entendu, on peut imaginer une évolution où les rôles des lignes $e_{1}$ et $e_{3}$ seraient intervertis).

De manière générale, les obstructions d'origine topologique au croisement des défauts entraînent ce qu'on pourrait appeler une rigidité topologique du système des défauts. L'ensemble des défauts se rapproche de la structure des polymères, avec condition de volume exclu. D'un point de vue pratique, cette rigidité du système des défauts devrait avoir des conséquences physiques observables dans les propriétés de réponse du milieu à des contraintes, éventuellement dans les écoulements, etc...

On espère avoir lancé ici suffisamment de prédictions théoriques pour stimuler chez les expérimentateurs le désir de les infirmer (ou de les confirmer).

\section{Bibliographie}

[1] La publication de cette lettre avait été ajournée dans l'espoir d'un proche succès dans cette voie. C'était sous-estimer le temps pour convaincre de l'intérêt du programme. Il est vrai aussi que pour le moment on ne voit pas de recette évidente. Je remercie le rapporteur pour sa suggestion de molécules avec une partie planaire conférant un caractère nématique planaire et une partie en bâtonnet du type rencontré dans les nématiques classiques.

[2] Toulouse, G., Kleman, M., J. Physique Lett. 37 (1976) 149; Toulouse, G., Bull. Soc. Fr. Physique, octobre 1976.
[3] On suppose, pour simplifier l'exemple, que les variables nucléaires suivent les variables orbitales.

[4] Dans le langage des spécialistes des défauts, cela veut dire qu'un défaut linéaire, correspondant à une rotation de $2 \pi$ du paramètre d'ordre sur un circuit entourant le défaut, ne peut pas échapper, alors que, dans le cas d'une rotation de $4 \pi$, il peut échapper.

[5] Michel, L., à paraître.

[6] Poenaru, V., article en préparation. 